\title{
Schnee- und Gletscherschmelze als Baustein der hydrologischen Abflussmodellierung
}

\section{Snow and glacier melt as a module for hydrological modelling}

von H. Holzmann, G. Koboltschnig, W. Schöner und L. Rauch

\section{Kurzfassung/Summary}

Die Modellierung hydrologischer Prozesse hat in den letzten Jahren verstärkt Einzug in operationelle Anwendungsbereiche gefunden. Die Ursachen liegen zum einen in der fortschreitenden Entwicklung leistungsstarker Datenerfassungsund Datenbearbeitungssysteme, in der Möglichkeit komplexe Einzugsgebietsgliederungen sowohl räumlich als auch zeitlich hoch aufzulösen und letztendlich auch an dem gesteigerten Bedarf vorausschauende Aussagen hinsichtlich der Abflussentwicklung bereitzustellen. Letztere sind unter dem Aspekt der Hochwasservorhersage und Warnung, aber auch der Niederwasservorhersage mit Einfluss auf die Energieerzeugung, die Binnenschifffahrt oder die Ökologie von Bedeutung. In den alpinen Regionen Österreichs spielt der Abflussbeitrag aus Schnee und Gletscherflächen eine große Rolle. Daher bildet dieser Anteil einen wichtigen Bestandteil der integrierten hydrologischen Modellierung. Im vorliegenden Beitrag wird auf verschiedene Verfahren der Schnee- und Gletscherschmelzmodellierung eingegangen, wobei der Schwerpunkt bei der Beschreibung von indexbasierten Modellen liegt. Weiters wird die Verschneidung von Schmelz- und Abflussmodellen angesprochen.

Since several years hydrological modelling has grown more important for operational applications. This is caused by the developing resources of data collection and processing systems, the increased capability of spatio-temporal re- solution of hydrological basins by geospatial techniques and by the growing demand of runoff prediction systems. The latter are strongly related on flood forecasts and warning systems but also on forecasts of droughts, hydro energy production or ecological developments. In alpine regions the contribution of melt by snow covered and glaciered areas is of high importance for an integrated approach of the entire hydrological processes. The current paper refers on different methods of melt modelling, where the focus is on index based concepts. Furthermore the linkage of melt and runoff formation is addressed.

\section{Einleitung}

Aufgrund der topographischen Situation Österreichs spielen bei der Beschreibung und Modellierung der Abflussverhältnisse die Schnee- und Eisschmelzprozesse eine wichtige Rolle. Sie prägen in zahlreichen Einzugsgebieten die jährliche Abflusscharakteristik und finden in den Klassifizierungen der nivalen bzw. nivo glazialen Abflussregime Berücksichtigung. Die Beobachtung von Schnee- und Gletscherprozessen und ihrer meteorologischen Randbedingungen ist aufgrund der exponierten Gegebenheiten mühsam und arbeitsintensiv. Dementsprechend eingeschränkt finden sich Beobachtungen und Datenreihen zu diesen Prozessen verglichen mit den hydro-meteorologischen Beobachtungen in niedriger gelegenen Bereichen.

Im Rahmen des Forschungsprojekts SNOWTRANS (Holzmann et al. 2008) der Österreichischen
Akademie der Wissenschaften hatten die Autoren die Möglichkeit zu einer Intensivierung hochalpiner Datenbeobachtungen. Dabei wurde sowohl auf bestehende Meßinfrastruktur zugegriffen wie auch eine Intensivierung der Beobachtungen vorgenommen (vgl. Schöner et al. 2009 in diesem Heft). Aus diesen Gründen wurde als Testgebiet das Einzugsgebiet des Goldbergkees Gletschers am Rauriser Sonnblick gewählt, da sich hier die Datenlage aufgrund des meteorologischen Obervatoriums Sonnblick, das von der Zentralanstalt für Meteorologie und Geodynamik betrieben wird, als ausgezeichnet erweist. Die gewonnenen Erkenntnisse aus dem Kleineinzugsgebiet wurden aber auch für andere Maßstabsbereiche in der Region Oberpinzgau angewandt und verifiziert (Vergleiche dazu den Beitrag von Koboltschnig et al., 2009 in diesem Heft).

Im o.g. Projekt gelangten unterschiedliche Modellkonzepte zur Anwendung. Koboltschnig (2007) beschreibt in seiner Dissertationsschrift die Verwendung des an der ETH Zürich entwickelten Modells PREVAH (Gurtz et al., 2003 ). In Michlmayr (2007) und Michlmayr et al. (2008) findet sich die Anwendung eines physikalisch basierten Ansatzes auf Basis des Energiebilanzverfahrens. Dabei wurde das Modell ALPINE-3D des schweizerischen WSL / SLF (vgl. Lehning et al., 2006) angewandt. Rauch (2007) verwendete einen, am Institut für Wasserwirtschaft, Hydrologie und konstruktiven Wasserbau (IWHW) entwickelten Programmansatz. Auf dieses Verfahren wird im aktuellen Beitrag näher eingegangen. 


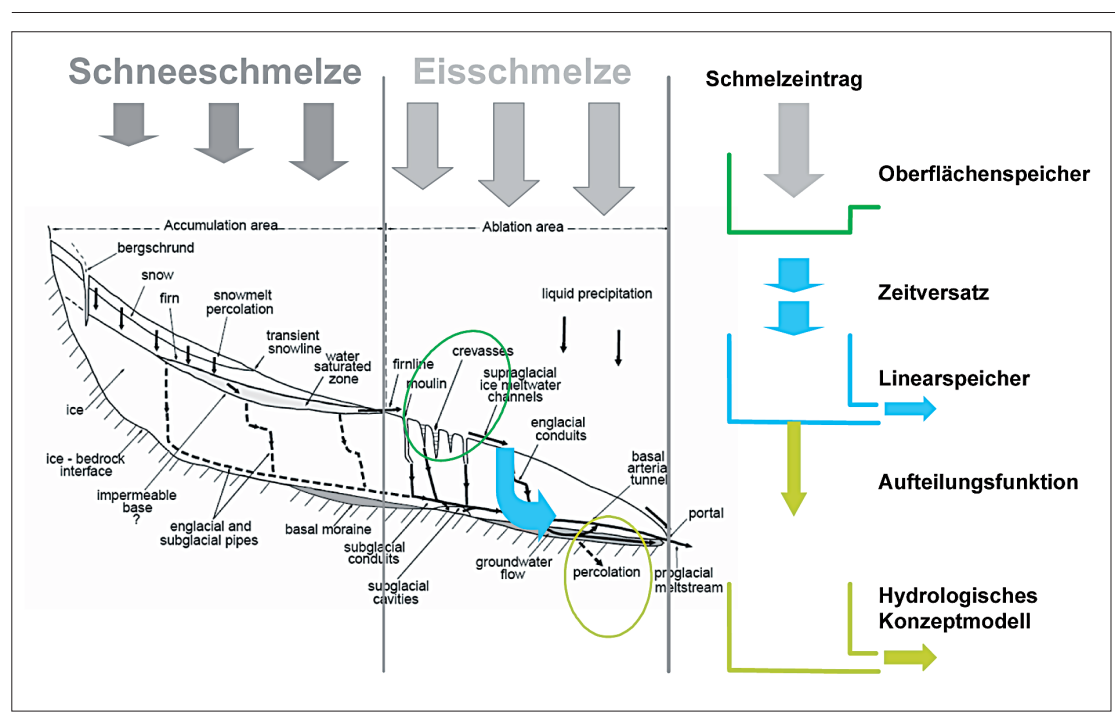

Abb. 1: Konzeptualisierung des Schmelzabflusses in einem Kleineinzugsgebiet.

\section{Datenbasis}

Als wichtigste meteorologische Kenngrößen gehen der Niederschlag, die Lufttemperatur und die Strahlung (Globalstrahlung) in die Modellierung ein. Daneben finden direkt oder indirekt Windgeschwindigkeit und -richtung sowie Dampfdruck Eingang. Diese Daten wurden vom Sonnblickobservatorium bereitgestellt. Daneben fanden Niederschlagsmessungen mittels Totalisatoren und temporär installierter Obrometer sowie Temperaturmesssonden Verwendung. Abflussbeobachtungen am Gebietsauslass des Kleineinzugsgebiets existieren für die Sommer und Herbstperioden seit 2002. Eine detaillierte Beschreibung sämtlicher Messungen findet sich in Schöner et. al. in diesem Heft.

Die für Schmelzprozesse bestimmenden Klimafaktoren Strahlung und Temperatur unterliegen sowohl einem jahreszeitlichen wie auch einem täglichen Zyklus. Ziel des Projekts war es auch die tägliche Variabilität der Prozesse zu untersuchen. Die angewandten Modellansätze sind für hohe zeitliche Auflösungen geeignet, es wurde in Stundenintervallen beobachtet und simuliert.

\section{Prozessbeschreibung und Modellkonzept}

Bei der hydrologischen Modellierung alpiner Regionen sind neben dem Niederschlag auch die Schmelzeinträge aus Schnee- und Eisflächen für die Abflussbildung zu berücksichtigen. Aufgrund der Abhängigkeiten von Lufttemperatur und Strahlung sind sie räumlich variabel und von der Seehöhe, der Neigung und Exposition bestimmt. Daraus ergeben sich bei kleinräumiger Betrachtung sehr variable Schmelzanteile und Ausaperungsmuster. Bei der Modellanwendung wurden unterschiedliche räumliche Diskretisierungen zur Berücksichtigung der Inputvariabilität angewandt. Bei der Modellierung mit PREVAH und ALPINE3D wurde eine Unterteilung in Hydrologisch ähnliche Teilgebiete (Hydrological Response Units HRU) durchgeführt. Diese Gliederung wurde sowohl für die Schmelzmodellierung wie auch für die Abflussagreggierung angewandt. Beim BOKU Modell erfolgte für die Schmelzberechnung eine Gliederung in Höhenzonen von 100 Metern, die Abflusstransformation erfolgte jedoch als flächenaggregierte Modellierung.

Abbildung 1 zeigt schematisch einen Schnitt durch einen Glet- scher. Die Gletscherfläche wird in einen Akkumulationsbereich und einen Zehrbereich untergliedert. Die Grenze bildet die Gleichgewichtslinie. Oberhalb der Gleichgewichtslinie erfolgt im langjährigen Mittel eine Anhäufung von Schnee, der später in Firn und Eis umgewandelt wird. Unterhalb erfolgt ein Abschmelzen der Eisdecke und der Abtransport des Schmelzwassers über oberflächliche Rinnen, Spalten und unterirdischen Abfluss an der Grenzschicht zwischen Eis und Fels. Falls der subglaziale Untergrund porös beschaffen ist, kann Schmelzwasser auch in den hydrogeologischen Untergrund infiltrieren und dort verzögert zum Abfluss gelangen. Dem Abschmelzen des Zehrbereichs wirkt das Fließen des Gletschers entgegen, der eine Massenverlagerung nach unten bewirkt.

Gletscherschmelze wird in den meisten Konzepten erst dann angenommen, wenn eine Ausaperung derüberlagerten Schneedeckegegeben ist. Aufgrund der unterschiedlichen meteorologischen Gegebenheiten gibt es große Unterschiede zwischen einzelnen Beobachtungsjahren. Im heißen Sommer 2003 erfolgte bei den meisten Gletschern in Österreich ein vollkommenes Abschmelzen der Schneedecke und daher ein verstärktes Schmelzen der Eismassen. Eine zuverlässige Gletscherschmelzmodellierung ist daher nur in Kombination mit einem räumlich gegliederten Schneeschmelzmodell gewährleistet.

Die Konzeptskizze im rechten Teil der Abbildung 1 zeigt die wichtigsten Komponenten der Gletscherschmelzmodellierung. Ein Speicherterm für den (1) Oberflächenspeicher berücksichtigt z.B. einen Rückhalt von Schmelzwasser in Gletscherspalten. Dieser Modul ist optional und von den glaziologischen Eigenschaften abhängig. Die Abflussprozesse im Eis erfolgen zumeist rascher und ungedämpfter als in der Schneedecke. Die Kon- 


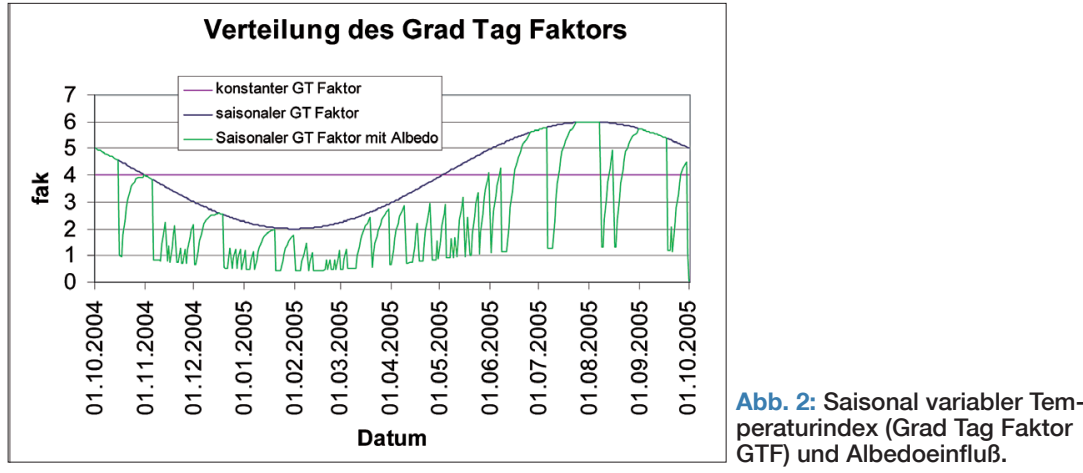

zentrationszeit zu einem Bezugspegel kann daher durch einen (2) Zeitverschiebungsterm berücksichtigt werden. Je nach Beschaffenheit der Gletscherhohlräume kann dieser Zeitversatz auch durch einen Einzellinearspeicher ersetzt werden oder eine Kombination aus beiden erfolgen. Gibt es unterhalb des Gletschers durchlässige Schichten (z. B. Grundmoräne), so ist der Anteil der infiltrierenden Schmelzrate zu quantifizieren ((3) Aufteilungsfunktion). Der unterirdische Abfluss wird in Analogie zu einem (4) hydrologischen Konzeptmodell je nach Komplexität als Einzellinearspeicher oder als Speicherkaskade simuliert. Die Schneeschmelzmodellierung erfolgt in ähnlicher Weise, allerdings mit unterschiedlicher Parameterisierung.

Am Institut fürWasserwirtschaft, Hydrologie und Konstruktiven Wasserbau (IWHW) der Universität für Bodenkultur Wien (BOKU) gelangten in vergangenen Studien unterschiedliche Modelltypen der Schneeschmelzmodellierung zur Anwendung. Aufgrund der unterschiedlichen Zielsetzungen erfolgten verschiedene raumzeitliche Gliederungen. Kling et al. (2006) führte eine Schmelzmodellierung auf Monatsbasis durch, wobei die räumliche Gliederung auf einen 1 x $1 \mathrm{~km}$ Raster erfolgte.

Holzmann \& Nachtnebel (2000) entwickelten im Zuge einer österreichweiten Abflussprognose für die Energiewirtschaft ein Schneeschmelzmodell, welches auf Ta- gesbasis und einer 500-Meter Höhenzonierung basierte. Stanzel et al. (2008) geben ein Beispiel der Schneeschmelzmodellierung in operationellen Vorhersagesystemen mit hoher raumzeitlicher Auflösung. In Habersack et al. (2006) wurden Schnee- und Eisschmelzmodelle auf Tagesbasis zur Abschätzung des Gletscheranteils am Gesamtabfluss eines mittleren Einzugsgebiets (Obere Salzach - Pegel Mittersill) angewandt und in Holzmann (2006) wurde dieser Ansatz mit höherer raumzeitlicher Auflösung versehen (Stundenwerte, 100-Meter Höhenzonierung).

Im Rahmen des Forschungsprojekts SNOWTRANS wurden Modellverbesserungen vorgenommen, welche die Albedo, die Expositionsverteilung innerhalb der Höhenzonen und den Kältegehalt berücksichtigen. In Rauch (2007) wurde diese Modellversion für das Goldbergkees angewandt und getestet. In Holzmann et al. (2008) ist die Anwendung für ein kleines Einzugsgebiet (Obersulzbach - Pegel Kees) und die Kopplung mit einem hydrologischen Konzeptmodell beschrieben.

\section{Methodik}

\subsection{Schnee- und Eisschmelze}

Der nachfolgend beschriebene Schmelzmodellansatz basiert auf einem Kombinierten Index Konzept, welches sowohl die Lufttemperatur und die Strahlung be- rücksichtigt. Beide Klimagrößen sind am Sonnblick beobachtet. Liegen keine direkten Strahlungsmessungen vor, so kann auch die potentielle Strahlung verwendet werden. Beim kombinierten Indexverfahren wird die Schneeschmelzrate als linear abhängige Größe von Lufttemperatur und Strahlung gesehen, welche durch die Indizes RI (Strahlungsindex) und TI (Temperaturindex) beschrieben ist. Wird $\alpha=1$ gewählt, so handelt es sich um das klassische Grad Tag Verfahren, bei $\alpha=0$ liegt ein Strahlungsindexansatz vor. Ähnliche gemischte Indexmodelle werden in Hock (1999) beschrieben.

$M_{i, k, j}=\frac{1}{d t}\left(\alpha \cdot \operatorname{TI}_{k} \cdot \operatorname{Temp}_{i, j}+(\alpha-1) \cdot R I \cdot \operatorname{Rad}_{i}\right)$

wobei:

M Schmelzrate ( $\mathrm{mm})$

TI Temperaturindex

RI Strahlungsindex

Temp positive Lufttemperatur-

Rad Globalstrahlung

k seasonaler Index

i zeilicher Index

j Höhenindex

a Gewichtungsfaktor

dt Zeiteinheit pro Tag

Gleichung 1 kann durch die nachfolgenden TermeAlbedofaktor albfak und Expositionsfaktor exfak erweitert werden. Der Albedofaktor berücksichtigt die geänderten Strahlungsreflexionsbedingungen bei Neuschnee. Die Berechnung erfolgt gemäß der nachfolgenden Gleichung:

$\operatorname{albfak}_{k}=1-\exp \left(\frac{- \text { idayalb }_{k}}{\text { fakk }}\right)$

wobei:

albfak Albedofaktor

idayalb Neuschneealter (in days)

fakk Rezessionsfaktor (default $=4.0 \mathrm{~d}$ )

$k \quad$ Höhenindex

Abbildung 2 zeigt eine Gegenüberstellung eines saisonal sinusförmig variierenden Temperaturindex 
Tab. 1: Die wichtigsten Schmelzparameter des kombinierten Modells

\begin{tabular}{lccc}
\hline Parameter & $\mathbf{2 0 0 3}$ & $\mathbf{2 0 0 4}$ & $\mathbf{2 0 0 5}$ \\
\hline$T I_{\text {Schnee }}$ & 3 & 2.85 & 4 \\
\hline$T I_{\text {Eis }}$ & 7.2 & 6.75 & 8 \\
\hline$R I_{\text {Schnee }}$ & 0.11 & 0.11 & 0.15 \\
\hline$R I_{\text {Eis }}$ & 0.25 & 0.15 & 0.2 \\
\hline Amplitude von $\mathrm{TI}$ & 1.1 & 1.5 & 2 \\
\hline Linearspeicherkoeffizient $_{\text {Schnee }}$ & 10 & 10 & 15 \\
\hline Linearspeicherkoeffizient $_{\text {Eis }}$ & 4 & 5 & 5 \\
\hline Linearspeicherkoeffizient $_{\text {Regen }}$ & 8 & 18 & 17 \\
\hline$\alpha$ (Gewichtung) $^{\text {Schmelztemperatur }}$ & 0.5 & 0.9 & 0.85 \\
\hline
\end{tabular}

mit und ohne Berücksichtigung des Albedoeffektes.

Im Schneeschmelzmodell werden Höhenzonen ideell als homogene Teilflächen behandelt. Tatsächlich existieren innerhalb der Zonen Bereiche unterschiedlicher Exposition und Hangneigungen mit differenziertem Energieeintrag und potentieller Schmelzrate. Durch topographische Analyse mittels GIS wurden die Variabilitäten hinsichtlich der Exposition analysiert und höhenzonenweise in 9 Klassen eingeteilt. Dabei werden nördliche Expositionen hinsichtlich der Schmelzkapazität geringer gewichtet als südliche. Diese Gewichtung variiert zwischen 0.8 (Norden) Ausrichtung) und 1.2 (Süden) und wird in 9 Ausrichtungsklassen abgestuft. Die Variationsbreite der Gewichtung wurde im Zuge der Kalibrierung festgelegt. Alternativ können Analysen des potentiellen Strahlungseintrags aus einem digitalen Geländemodell eingehen. Die Berechnung ergibt sich wie folgt:

$$
\text { exfak }=\frac{1}{100} \cdot \sum_{k=1}^{9} \text { exarea }_{k} \cdot f f_{k}
$$

wobei:

exfak Expositionsfaktor der Höhenzone

exarea Flächenanteil der 9 Expositionsklassen (in Prozent)

ff Gewichtungsfaktor der 9
Expositionsklassen

$k \quad$ Index der Expositionsklasse (1 to 9)

Zusätzlich wurde ein Kältegehaltsfaktor eingeführt, welcher berücksichtigt, dass für das Einsetzen der Schneeschmelze ein bestimmter Energieeintrag notwendig ist, um eine Schneemetamorphose von Kaltschnee $\mathrm{zu}$ Naßschnee zu ermöglichen. Dieser Energieeintrag wird vereinfacht durch die Tagestemperatursumme der letzten $n$ Tage (default 21 Tage) charakterisiert. Er muss positiv sein, um Schmelze zu ermöglichen. Die nachfolgende Gleichung berücksichtigt auch die lineare Extrapolation der Lufttemperatur einer Meßstation auf die entsprechende Höhenzone mittels hypsometrischen Temperaturgradient.

$$
c c f a k_{j}=\sum_{k=1}^{-n} \text { tempref }_{k}+\left(\text { hypsgrad } \cdot\left(\text { gebalt }_{j}-\text { altref }\right)\right)
$$

wobei:

ccfak Kältegehaltsfaktor der Höhenzone j

tempref Stationstemperatur

hypsgrad hypsometrischer Temperaturgradient

gebalt Mittl. Seehöhe der Zone j

altref Seehöhe der Temperaturstation

\subsection{Abflusstransformation}

Durch Akkumulation der flächengewichteten Schmelzraten der einzelnen Höhenzonen wird die Gesamtschmelze des Einzugsgebiets berechnet. Für den Vergleich dieser Schmelze mit dem beobachteten Abfluss am Bezugspegel ist eine Flächentransformation $\mathrm{zu}$ berücksichtigen. Die kann zum einen durch Annahme eines Zeitverzögerungsterms erfolgen oder durch Transformation mittels Einzellinearspeicher. Durch das Fehlen eines nennenswerten unterirdischen Abflussanteils erscheint für dieses Testeinzugsgebiet die o.g. Vorgangsweise als gerechtfertigt. Dabei erfolgt die Transformation von Regen, Schneeschmelz- und Gletscherschmelzanteil in drei separaten Linearspeicher mit differenzierter Parameterisierung.

Die Transformation der Gletscherschmelze erfolgt aufgrund der eingeschränkten Speicherkapazität relativ rasch. Hier kann der Abfluss durch einen Oberflächenspeicher (repräsentiert die Wasserspeicherung an oberflächigen Rillen oder Spalten) und einem Zeitversatz gut wiedergegeben werden. Regen auf schneefreier Fläche wird hinreichend über einen Einzellinearspeicher beschrieben. Abflussverluste infolge Infiltration sind zu vernachlässigen. Bei Schneeschmelzabfluss erfolgt die größte zeitliche Verzögerung aufgrund der Speicherkapazität in der Schneedecke. Hier sind die Speicherkoeffizienten größer zu wählen.

\section{Ergebnisse}

Rauch (2007) verglich in seiner Diplomarbeit das Grad-Tag-Verfahren (Temperaturindexverfahren), das Strahlungsindexverfahren und das Kombinierte Indexverfahren der in Gleichung 1 beschriebenen Form. Mit letzterem Verfahren konnten die besten Ergebnisse erzielt werden. In Folge beziehen 


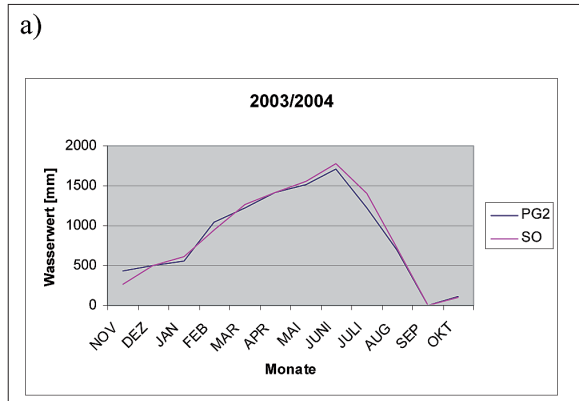

b)

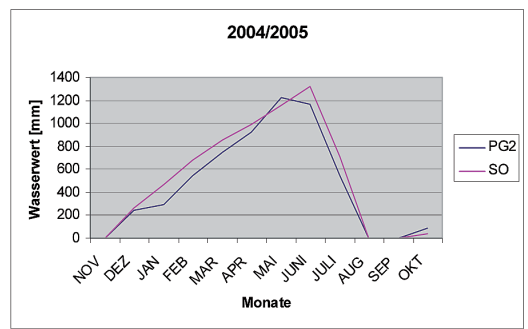

c)

d)
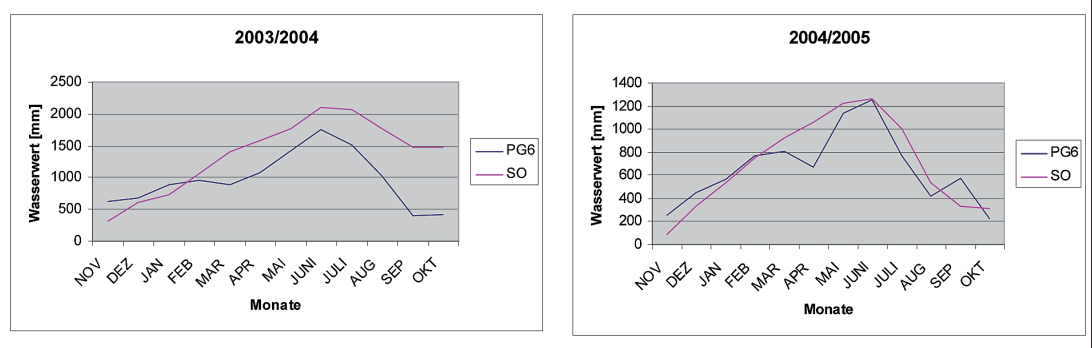

Abb. 3: Vergleich des beobachteten (blau) und simulierten (rot) Schneewasserwertes der Schneepegel PG2 a), b) (2650 m Sh.) und PG6 c), d) (3050 m Sh.) (Rauch, 2007).

a)

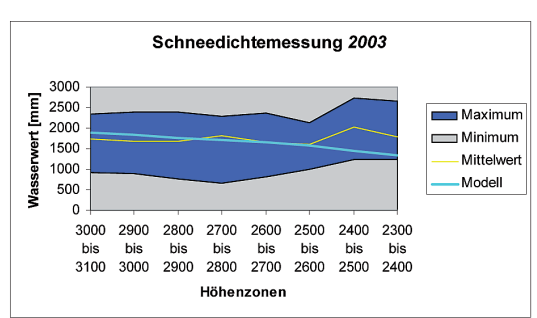

c)

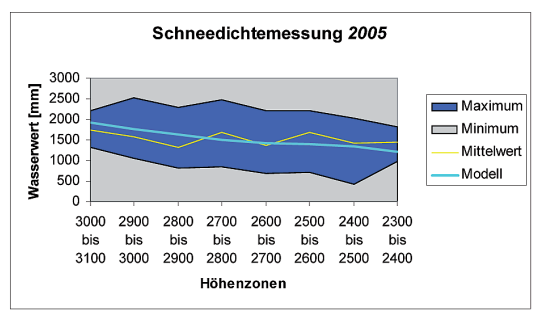

Abb. 4: Vergleich simulierter (hellblau) und beobachteter Schneewasserwerte (aus Rauch, 2007)

sich die Erläuterungen auf das Kombinierte Verfahren. Die durch Kalibrierung gewonnenen Modellparameter sind in Tabelle 1 angeführt. Die Prüfung des Modells erfolgte anhand der Abflussbeobachtungen und anhand Schneepegeldaten, aus denen der Schneewasserwert durch ergän- b)

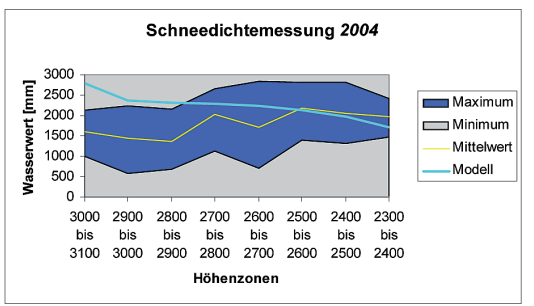

zende Dichtemessungen errechnet wurde. Die errechneten Korrelationskoeffizienten der Stundenwerte waren 0.85 für die Jahre 2003 und 2004 und 0.89 für das Jahr 2005.

Abbildung 3 zeigt den Vergleich von simuliertem und beobachtetem Schneewasserwert der Winterperioden 03/04 sowie 04/05. Die
Schneepegel liegen in $2650 \mathrm{~m}$ (PG2) und $3050 \mathrm{~m}$ Seehöhe (PG6). Die Übereinstimmung des Verlaufs für den unteren Pegel ist sehr gut. Sowohl die Akkumulations- wie auch die Schmelzphase sind gut wiedergegeben. Am Pegel 06 wird im Jahr 03/04 die Schneeakkumulation überschätzt, die Schmelzintensität unterschätzt. Als Grund wird die nicht erfasste Schneedrift durch Wind angesehen.

Im Mai eines jeden Jahres erfolgt zumeist ein umfangreiches Monitoringprogramm der Schneeakkumulation im Gebiet, in dessen Rahmen an mehreren Standorten Schneedichtemessungen durchgeführt werden (Vergleiche Schöner et al., 2004). In Abbildung 4 ist die Meßbreite der Beobachtungen den simulierten Werten gegenübergestellt. Für die Jahre 2003 und 2005 liegen die Simulationswerte innerhalb der beobachteten Variationsbreite. Im Jahr 2004 sind die Schneewasserwerte der höheren Zonen (2800 bis 3000 m Sh.) im Modell überschätzt. Dies ist wiederum auf das Fehlen eines Schneedriftmoduls zurückzuführen.

Der Vergleich der Abflussganglinien (vgl. Abbildung 5) zeigt eine gute Übereinstimmung zwischen beobachteten und simulierten $\mathrm{Ab}$ flüssen. Die Spitzen sind geringfügig unterschätzt, der Tagesgang ist jedoch recht gut wiedergegeben. Rauch (2007) weist die Güte (Korrelationsmaß) der verschiedenen Indexverfahren (Temperaturindex, Strahlungsindex, Gemischter Index) aus. Seine Ergebnisse sind in der nachfolgenden Tabelle 2 aufgelistet.

Für das kleine, hoch gelegene Einzugsgebiet des Goldberkees konnte mit dem, in Abbildung 1 dargestellten Modellkonzept ein gutes Ergebnis erzielt werden, wobei auf die Koppelung mit einem hydrologischen Modell verzichtet wurde und nur ein Einzellinearspeicher berücksichtigt wurde. Bei größeren Einzugsgebieten spielt der unterir- 


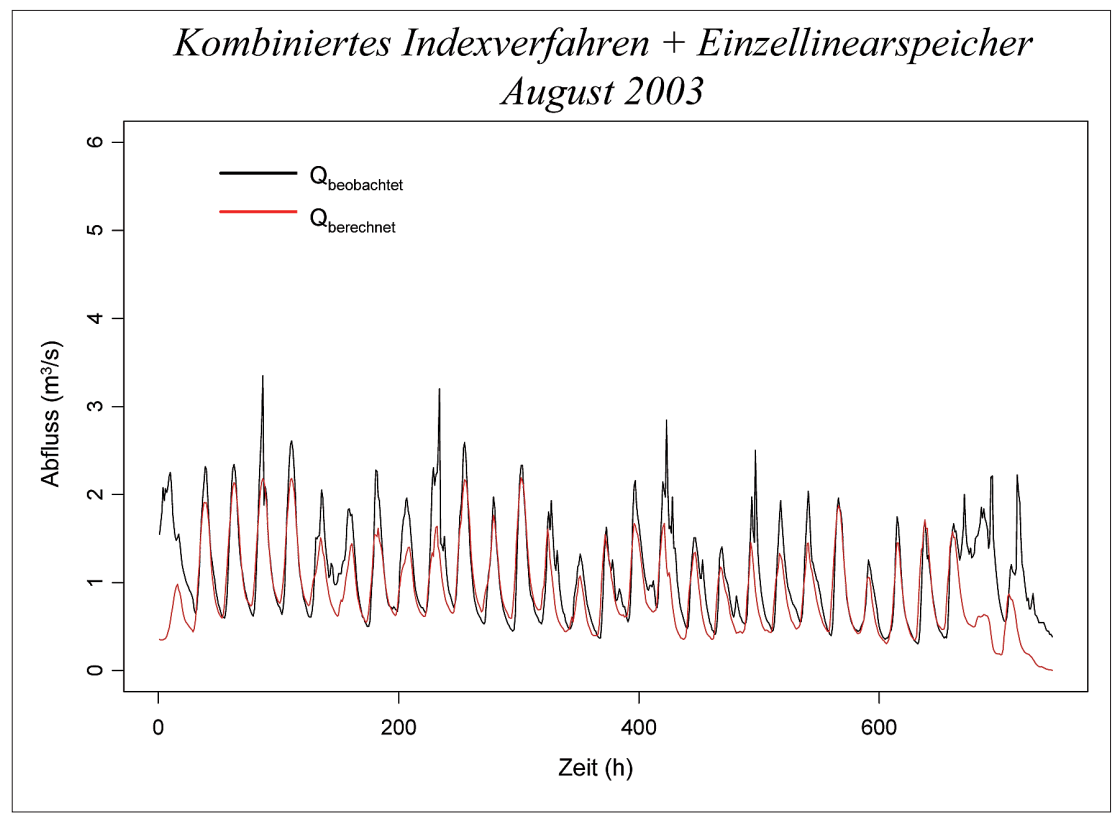

Abb. 5: Vergleich von beobachtetem und simuliertem Abfluß Pegel Goldbergkees (aus Holzmann, 2007).

Tab. 2: Gütemaß (Korrelation) zwischen simulierten und beobachteten Abflüssen

\begin{tabular}{lccc}
\hline Methode & $\mathbf{2 0 0 3}$ & $\mathbf{2 0 0 4}$ & $\mathbf{2 0 0 5}$ \\
\hline Temperaturindex & 0.79 & 0.86 & 0.87 \\
\hline Strahlungsindex & 0.82 & 0.71 & 0.74 \\
\hline Kombinierter Index & 0.85 & 0.85 & 0.89 \\
\hline
\end{tabular}

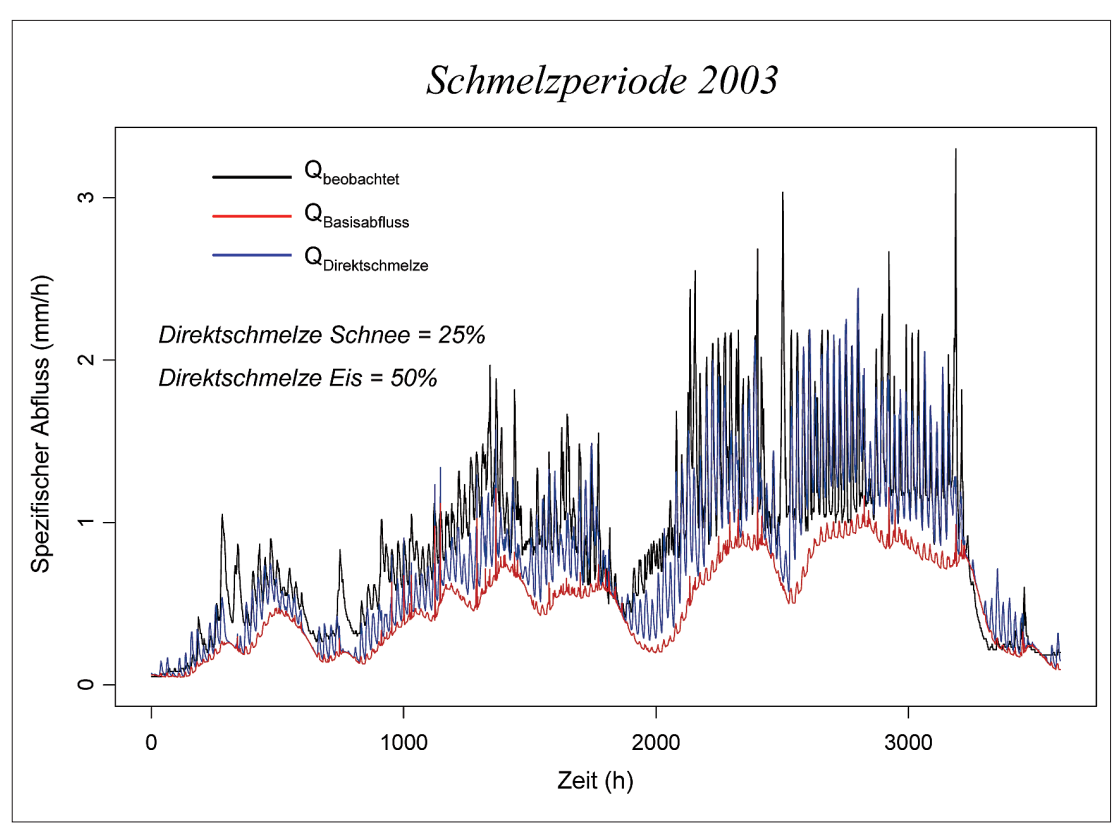

Abb. 6: Vergleich zwischen beobachteten und simulierten Abflüssen des Pegels Kees/Obersulzbach (aus Holzmann, 2008). dische Abfluss bei der Abflussentstehung eine große Rolle. Daher wurde für diesen Maßstabsbereich ein konzeptuelles Modell wie in Holzmann et al. (2002) beschrieben gekoppelt. Abbildung 6 zeigt die unterschiedlichen Abflussanteile. Rot dargestellt ist die unterirdische Abflusskomponente. Diese wird vom direkten Abfluss aus der Gletscherschmelze und dem Schnee überlagert. Dabei wird mittels der Aufteilungsfunktion (vgl. Abb. 1) $75 \%$ der Schneeschmelze und $50 \%$ der Gletscherschmelze in den Untergrund transformiert. Der Gesamtabfluss wird am Beginn der Schmelzperiode etwas unterschätzt. Dies lässt auf eine Unterschätzung des akkumlierten Winterniederschlags schließen.

\section{Ausblick}

Schnee- und Gletscherschmelze sind wesentlich Teilprozesse der Abflussbildung im hochalpinen Bereich. Ihre zuverlässige Quantifizierung ist eine wesentliche Voraussetzung für die Abflussmodellierung im Rahmen hydrologischer Untersuchungen oder operationeller Abflussvorhersagen. Dieser Beitrag zeigt, dass mit einfachen indexbasierten Modellen durchaus zufriedenstellende Ergebnisse zu erzielen sind. Bei Tageswertmodellen genügt mitunter die Verwendung von Temperaturindexverfahren. Bei zeitlich höher aufgelöster Betrachtung erweisen sich kombinierte Ansätze unter Verwendung von Strahlungsdaten als besser geeignet, da sie die Variabilität im Tagesverlauf besser zum Ausdruck bringen. Die räumliche Diskretisierung soll zumindest eine gute Höhenauflösung gewährleisten (100-200 Meter) um die Temperaturabhängigkeit der Schmelze zu berücksichtigen. Sollen flächenhafte Ausaperungsmuster wiedergegeben werden, so ist eine Gliederung in Rasterzellen oder in HRUs unter Berücksichtigung der Exposition zu empfehlen. Durch Kombination mit Fernerkun- 
dungsverfahren lassen sich ebenfalls verbesserte räumliche Auflösungen erzielen (vergleiche Kaiser et al. in diesem Heft.)

Dennoch zeigen sich unabhängig vom Modelltyp oftmals Schwierigkeiten in der Modellierung die nachfolgend aufgezählt werden. Die Güte der Schmelzmodellierung hängt in hohem Maße von der zuverlässigen Schätzung der Niederschlagsverteilung im Gebiet ab. Gerade in hochalpinen Regionen sind meteorologische Stationen selten. Zusätzlich sind die Messdaten durch äußere Effekte (z.B. Windeinfluss) fehlerhaft. Daher zeigen sich im Zuge der Schmelzmodellierung oft Bilanzfehler, die durch Unterschätzung des gefallenen Niederschlags (Regen oder
Schnee) bedingt sind. Notwendige Niederschlagskorrekturen bis $\mathrm{zu}$ $50 \%$ sind daher nicht selten. In hochalpinen Lagen spielen auch Schneeverfrachtungen durch Wind und Lawinen eine große Rolle. Gerade in den kammnahen Bereichen wird der Schnee abgeweht und die Modelle überschätzen daher die Akkumulation in diesen Höhenlagen. Mott (2007) untersuchte in ihrer Diplomarbeit die Möglichkeit der Verwendung dynamischer Windfelder zur Beschreibung der Schneeverfrachtung. Ein hohes Potential in der Verbesserung der räumlich verteilten Schneeschmelzmodellierung versprechen auch Fernerkundungsdaten, die zusehends in besserer zeitlicher und räumlicher Auflösung aufgenommen werden. Sie liefern eine weitere Validierungsmöglichkeit der jeweiligen Modellansätze.

\section{Danksagung}

Die Autoren bedanken sich bei der Österreichischen Akademie der Wissenschaften für die Finanzierung des Projekts SNOWTRANS (Regionalisation of snow- and ice melt processes in the Hohe Tauern mountains in Austria) im Rahmen der Hydrologie Österreichs (HÖ29).

\section{Korrespondenz:}

Ao. Univ.-Prof. DI Dr. Hubert HOLZMANN Institut für Wasserwirtschaft, Hydrologie und Konstruktiven Wasserbau Universität für Bodenkultur Wien Muthgasse 18, 1190 Wien

E-Mail: hubert.holzmann@boku.ac.at

\section{LITERATUR}

Gurtz J, Zappa M, Jasper K, Lang H, Verbunt M, Badoux A, Vitvar T (2003) A Comparative Study in Modelling Runoff and its Components in Two Mountainous Catchments. Hydrological Processes 17: 297-311. DO : 10.1002/hyp.1125

Habersack HM, Krapesch G, Holzmann H, Koboltschnig G (2006) Hitze- und Dürrefolgen für die Wasserschnig G (2006) Hitze- und Durrefold

Hock R (1999) A distributed temperature index iceand snowmelt model including potential direct solar radiation. Journal of Glaciology 45/149, 101-111.

Holzmann H (2006) Benutzerbeschreibung eines höhenverteilten Schneeschmelzmodells. IWHW-BOKU, Eingenverlag.

Holzmann H, Nachtnebel HP (2002) Abflussprognose für mittelgroße Einzugsgebiete - Methodik und Anwendungen.. Österr. Wasser- und Abfallwirtschaft, 54 9-10, 142-153.Hynek, B. (2006): Energiebilanz des Goldbergkees während des Sommers 2003. Diplomarbeit an Institut für Meteorologie der Universität Wien, Austria.

Holzmann H, Schöner W, Koboltschnig G, Kroisleitner Ch, Hynek B, Mott R, Michlmayer G, Schneide W, Kaiser G, Vollmann M, Rauch L (2008) Modeling and exterpolation of snow and ice melt processes in alpine environments. Final report of SNOWTRANS project. Austrian Academy of Sciences online publication. ISBN:
978-3-7001-3987-4, (http://epub.oeaw.ac.at/3987-4inhalt).

Hynek B, Schöner W (2004) Massenhaushalt 2002/2003 der Gletscher in der Goldberggruppe. 101-102. Jahresbericht des Sonnblick-Vereines: 1-15. Eigenverlag der Zentralanstalt für Meteorologie und Eigenverlag der Zen

Kling H, Fürst J, Nachtnebel HP (2006) Seasonal, Kling H, Fürst J, Nachtnebel HP (2006) Seasona spatially distributed modelling of accumulation and meting of snow for computing runoff in a long-term, largebasin water balance model. Hydrological Processes, 20

Koboltschnig G (2007) Multivalidation approach of hydrological snow and ice melt models in high alpine, glaciated catchments. Doctoral thesis at the BOKU uniersity, Vienna.

Koboltschnig G, Schöner W, Zappa M, Kroisleitne Ch, Holzmann H (2008) Runoff modelling of the glacierized Alpine Upper Salzach basin (Austria): multi-criteria result validation. Hydrological Processes, Vol. 22 / 19, pp 3950-3964.

Lehning M, Voelksch I, Gustafsson D, Nguyen T, Staehli M, and Zappa M (2006) A detailed model of mountain surface processes and its application to snow hydrology. Hydrological Processes. 20: 2111 - 2128.

Michlmayr G (2007) Simulation von Schnee- und Eis- schmelzprozessen in hochalpinem Raum. Diplomarbeit an der Universität für Bodenkultur Wien.

Michlmayr G, Lehning M, Koboltschnig G, Holzmann H, Zappa M, Mott R, Schöner W (2008) Application of the Alpine 3D model for glacier mass balan ( gical Processes, Vol. 22 / 19, pp 3941-3949.

Mott R (2007) Mott R (2007) Modellierung der zeitlichen Entwicklung und răumlichen Verteilung einer saisonalen Schnee decke vergletscher Gebiete (Sonnblick) mit dem phy sikalisch basierten Model A OKU University Vienna.

Rauch L (2007) Schneeschmelzmodellierung mit indexbasierten Modellen. Diplomarbeit an der Universität für Bodenkultur.

Schöner W, Hynek B (2006) Massenhaushalt 2004/2005 der Gletscher in der Goldberggruppe: Goldbergkees, Kleinfleißkees, Wurtenkees. Jährlicher Berich der Klimaabteilung der ZAMG Wien.

Stanzel Ph, Haberl U, Nachtnebel HP (2008) Modelling snow accumulation and snow melt in a continuous hydrological model for real-time flood forecasting . In Brilly, M. and Šraj, M. (Eds.), Conference Papers, XXIVth Conference of the Danubian Countries, 2-4 June 2008 Bled; ISBN: 978-961-91090-2-1 